\title{
Intérêts et signification écophysiologique de l'estimation de la biomasse et de l'activité photosynthétique de diverses fractions de taille phytoplanctonique en milieu lacustre eutrophe \\ Interests and ecophysiological significance of the estimation of biomass and photosynthetic activity in three phytoplanktonic size fractions in an eutrophic lake
}

\author{
L. Aleya et J. Devaux
}

Volume 2, numéro 3, 1989

URI : https://id.erudit.org/iderudit/705035ar

DOI : https://doi.org/10.7202/705035ar

Aller au sommaire du numéro

\section{Éditeur(s)}

Université du Québec - INRS-Eau, Terre et Environnement (INRS-ETE)

ISSN

0992-7158 (imprimé)

1718-8598 (numérique)

\section{Découvrir la revue}

Citer cet article

Aleya, L. \& Devaux, J. (1989). Intérêts et signification écophysiologique de l'estimation de la biomasse et de l'activité photosynthétique de diverses fractions de taille phytoplanctonique en milieu lacustre eutrophe. Revue des sciences de l'eau / Journal of Water Science, 2(3), 353-372.

https://doi.org/10.7202/705035ar
Résumé de l'article

L'évolution saisonnière de la biomasse phytoplanctonique (estimée par les biovolumes après dénombrements cellulaires et par le dosage de la chlorophylle $a$ ), de la production primaire et des coefficients d'activité (P/"BComptage" et P/"BChlorophylle") a été étudiée, par classes de taille, de mars à novembre 1985 dans un lac eutrophe (lac d'Aydat, Massif Central).

Il apparaît que la fraction de taille $12-45 \mu \mathrm{m}$ contribue pour environ $50 \%$ à la biomasse phytoplanctonique totale quelque soit le descripteur de ta biomasse utilisé et pour $65 \%$ à l'activité photosynthétique totale, l'importance quantitative de cette fraction de taille se traduit au niveau des phénomènes d'extinction de la lumière par une relation inverse et hautement significative entre concentrations en chlorophylle a associée à cette classe et les profondeurs de disparition du disque de Secchi (Zs).

Si les corrélations linéaires établies entre la production primaire et les rapports surface/volume (S/V) sont significatives au sein de chaque classe, confirmant ainsi l'existence d'une relation directe entre ces 2 paramètres, les valeurs moyennes de $\mathrm{P} / \mathrm{B}$ de la fraction de taille $<12 \mu \mathrm{m}$ sont très différentes selon le mode d'expression de la biomasse. L'explication de cette divergence est à rattacher aux divers biais méthodologiques introduits dans les estimations de biomasse retenues dans notre étude. D'une part, la sous estimation de la fraction nanoplanctonique par la technique des dénombrements cellulaires entraîne par voie de conséquence une surestimation des valeurs de $\mathrm{P} / \mathrm{B}$ "Comptage" attribuées à la fraction $<12 \mu \mathrm{m}$. D'autre part, il est reconnu que les teneurs en pigments sont, par unité de volume, supérieures pour la fraction nanoplanctonique que pour les cellules de grande taille. L'adoption d'un facteur unique de conversion entraîne donc une surestimation de ta biomasse de cette fraction. 


\section{Intérêts et signification écophysiologique de l'estimation de la biomasse et de l'activité photosynthétique de diverses fractions de taille phytoplanctonique en milieu lacustre eutrophe}

Interests and ecophysiological significance of the estimation of biomass and photosynthetic activity in three phytoplanktonic size fractions in an eutrophic lake

L'évolution saisonnière de la biomasse phytoplanctonique (estimée par. Les biovolumes après dénombrements cellulaires et par le dosage de la chlorophylle $a$ ), de la production primaire et des coefficients d'activité ( $P /$ "BComptage" et P/"BChlorophylle") a été étudiée, par classes de taille, de mars à novembre 1985 dans un lac eutrophe (lac d'Aydat, Massif Central).

Il apparaît que la fraction de taille 12-45 $\mu \mathrm{m}$ contribue pour environ $50 \%$ à la biomasse phytoplanctonique totale quelque soit le descripteur de la biomasse utilisé et pour $65 \%$ à l'activité photosynthétique totale. L'importance quantitative de cette fraction de taille se traduit au niveau des phénomenes d'extinction de la lumière par une relation inverse et hautement significative entre concentrations en chlorophylle a associée à cette classe et les profondeurs de disparition du disque de Secchi (zs).

Si les corrélations linéaires établies entre la production primaire et les rapports surface/volume (S/V) sont significatives au sein de chaque classe, confirmant ainsi l'existence d'une relation directe entre ces 2 paramètres, les valeurs moyennes de $P / B$ de La fraction de taille $<12$ um sont très différentes selon le mode d'expression de la biomasse. L'explication de cette divergence est à rattacher aux divers biais méthodologiques introduits dans les estimatiors de biomasse retenues dans notre étude. D'une part, la sous estimation de

(1) Laboratoire de zoologie et protistologie, U.A. C.N.R.S. : 138 , GRECO lacs : 81, E3177 Aubiere Cédex. France. 
La fraction nanoplanctonique par la technique des dénombrements cellulaires entraîne par voie de conséquence une surestimation des valeurs de $P / B^{\prime \prime C o m p t a g e " ~ a t t r i b u e ́ e s ~ a ̀ ~ l a ~}$ fraction < $12 \mu \mathrm{m}$. D'autre part, il est reconnu que les teneurs en pigments sont, par unité de volume, supérieures pour la fraction nanoplanctonique que pour les cellules de grande taille. L'adoption d'un facteur unique de conversion entraine donc une surestimation de la biomasse de cette fraction.

Mots clés : fraction de taille, $P / B$, production primaire, bionasse, chlorophylle a, lac.

SUMMARY

The seasonal distribution of phytoplanktonic biomass (estimated by biovolumes after cell counts and by chlorophyll $a$ concertrations), primary production and primary production to biomass ratios ( $P / G^{\prime \prime C o u n t s " ~ a n d ~} P / B^{\prime \prime C h L o r o p h y(L ~} a^{\prime \prime}$ ) was studied by size fractionation in a eutrophic lake (lake Aydat, Massif (entral).

It appears that the 12-45 um size fraction contributes about $50 \%$ of the total phytoplanktonic biomass, regardless of what descriptor of the biomass is used and up to $65 \%$ of the total photosynthetic activity. Clearly, the quartitative predominance of this fraction affects the water transparency ( $Z s$ ) through the higly significant negative correlation obtained between $\mathrm{Zs}$ and the 12-45 $\mathrm{m}$ chlorophyll a size fraction.

The highly significant linear correlations obtained between the primary production of each fraction and surface to volume ratios (S/V) confirm the direct link between these two parameters. The mean values relative to the $P / B$ oi the $<12 \mathrm{\mu m}$ fraction depend largely on the method used for the biomass estimates. First, the underestimation of the nanoplanktonic fraction by cell counts leads to an over-estimation of the $<12 \mu \mathrm{m} P / B^{\prime \prime C}$ Counts" fraction. Second, it is well known that chlorophyll a concentrations per unit volume are higher for nanoplankton than for larger cells. Thus, the use of the same biomass conversion factor fcr all the fractions, overestimates the nanoplankton biomass.

Key-words : size fractionation, $P / B$, primary production, biomass, chlorophyle a, lake.

1 - INTRODUCTION

Le fractionnement en classe de taille des communautés phytoplanctoniques a fait l'objet de nombreux travaux tant en milieu lacustre (KALFF, 1972 ; MUNAWAR et MUNAWAR, 1975 ; PLATT et DENMAN, 1977 ; SIEBURTH et al., 1978 ; RAI, 1982 ; ELSER et al., 1986 ; MUNAWAR et MUNAWAR, 1986 ; TAKAMURA et al., 1986, MUNAWAR et al., 1988) que marin 
(MALONE, 1971 ; CARON, 1983 ; REID, 1983 ; HEWES et HOLM-HANSEN, 1983 ; MARGALEF et ESTRADA, 1987). L'analyse du peuplement algal pris dans sa globalité, peut masquer d'importantes différences, notamment au niveau des caractéristiques écophysiologiques des diverses espèces pxésentes. Plusieurs études réalisées en milieux maxins et lacustres ont montré que des phénomènes tels que la respiration (LAWS, 1975 ; SPODNIEWSKA, 1978, 1979 ; RAI, 1988), le recyclage des nutriments (PAERL et MACKENZIE, 1977 ; HERBLAND et BOUTEILLER, 1981 ; PETERS, 1983), les quotas cellulaixes en éléments nutritifs (SHUTER, 1978 ; SMITH et KALFF, 1982) le taux de sédimentation (SMAYDA, 1970; BIENFANG, 1985) l'activité photosynthétique et le taux de croissance (BANSE, 1976 ; TAGUCHI, 1976 ; MALONE, 1980 ; REYNOLDS, 1984 ; CRAIG, 1984 ; MURPHY et HAUGEN, 1985), la toxicité (MUNAWAR et al., 1988) sont largement dépendants du spectre de taille des cellules phytoplanctoniques. Par ailleurs, les travaux recents relatifs aux relations trophiques entre producteurs et consommateurs ont mis en évidence une sélectivité du broutage par les espèces zooplanctoniques en fonction des capacités de filtration, elles-mêmes directement liées à la taille des cellules phytoplanctoniques disponibles (STEELE et FROST, 1977 ; MAC CAULEY et KALFF, 1980 ; LYNCH et SHAPIRO, 1981 ; POURRIOT, 1982 ; BERGQUIST et al, 1985 ; ELSER et al., 1986 ; STOCKNER et ANTIA, 1986). Enfin, la prédation exercée sur les algues par les poissons (DRENNER et al., 1984 ; SPRULES et MUNAWAR, 1986 ) et les ciliés (GATES et LEWG, 1984 ; FINLAY et BERNINGER, 1984 ; ADMIRAL et VENEKAMP, 1986) dépend elie aussi des variations du spectre de taille du phytoplancton.

Dans le cadre d'une étude globale des communautés planctoniques d'un petit lac eutrcphe du Massif-Central français (lac d'Aydat), les évolutions de la biomasse phytoplanctonique, de la production primaire et du coefficient d'activité $(P / B)$ de trois classes de taille phytoplanctoniques : $1-12 \mu \mathrm{m} ; 12-45 \mu \mathrm{m}$ et $45-160 \mu \mathrm{m}$ ont été suivies de mars à novembre 1985 .

\section{2 - Matériel et méthodes}

\section{2-1 Le site}

Le lac d'Aydat est situé dans le Massif Central (dépaxtement du Puyde-Dôme, France). Il s'agit d'un lac dimictique à caractère eutrophe (MILLERIOUX, 1976 ; ALEYA et $a l ., 1988 a$ ) dont les caractéristiques morphométriques et physico-chimiques sont représentées dans le tableau 1.

\section{2-2 Echantillonnage}

Ia présente étude a été réalisée du 26 mars au 26 novembre 1985 à partir de compagnes hebdomadaires de prélèvements. Les échartillons ont été récoltés à la verticale du point central (profondeur $-14,5 \mathrm{~m}$ ) aux niveaux suivants : (près de la surface, $-1,-2,-4,-7,-10$ et $-14 \mathrm{~m}$ ) à I'aide d'une bouteille de type Van Dorn en PVC noir, modifiée pour prélever horizontalement en raison de l'importante stratification des eaux. 
Tableari 1.- Caractéristiques morphométriques (a) et physico-chimiques (b) du lac d'Aydat.

Table 1.- Morphometric characteristics (a) and abiotic parameters (b) of Aydat Lake.

$\begin{array}{lc}\text { Altitude du plan d'eau } & 825 \mathrm{~m} \\ \text { Superficie (A) } & 60,3 \times 10^{4} \mathrm{~m}^{2} \\ \text { Volune } & 4,14 \times 10^{6} \mathrm{~m}^{3} \\ \text { Longueur } & 175 \mathrm{~m} \\ \text { Largeur maximale } & 456 \mathrm{~m} \\ \text { Profondeur maximale (Zm) } & 15,50 \mathrm{~m} \\ \text { Profondelir moyenne (Z) } & 7,40 \mathrm{~m} \\ \text { Z/Zm } & 0,48 \\ \text { Surface du bassin versant } & 30 \times 10^{6} \mathrm{~m}^{2} \\ \text { Creux du lac (Zm/ } \sqrt{\mathrm{A}}) & 0,020\end{array}$

\begin{tabular}{|c|c|c|c|c|}
\hline & Unitês & Moyennes & Ecart-types & \\
\hline Rêsistivitê & $\mu S^{-1}$ & 9013,87 & 451,42 & \\
\hline $\mathrm{O}_{2}$ & $\mathrm{mg} \cdot 1^{-1}$ & 8,16 & 4,04 & \\
\hline$t^{0}$ & ${ }^{\circ} \mathrm{C}$ & 12,20 & 6,20 & \\
\hline Secchi & $\mathrm{m}$ & 1,70 & 0,26 & \\
\hline $\mathrm{NO}_{3}{ }^{-}$ & $\operatorname{mg} .1^{-1}$ & 1,02 & 1,24 & (b) \\
\hline $\mathrm{NO}_{2}^{-}$ & " & 0,38 & 0,26 & \\
\hline $\mathrm{NH}_{4}^{+}$ & $"$ & 0,09 & 0.06 & \\
\hline $\mathrm{PO}_{4}{ }^{3-}$ & $"$ & 0.07 & 0,05 & \\
\hline $\mathrm{SIO}_{2}$ & $"$ & 14,59 & 2,41 & \\
\hline
\end{tabular}

\section{2-3 Estimation de biomasse}

Deux méthodes ont été retenues :

a) Calcul des biovolumes par la technique de LOHMAN (1908) appliquée aux dénombrements cellulaires effectués au microscope inversé selon la méthode d'UTERMOHL (1958) mođifiêe par LEGENDRE et WATT (1971-1972).

La répartition des différentes espèces au sein de chacune des classes đe taille retenues ici a été réalisée en considérant la longueur du plus grand axe des cellules ou des colonies (ALEYA et DEVAUX, 1989).

b) Dosage de ta chlorophylle $a$ : selon un protocole expérimental dérivé de celui préconisé par SCOR-UNESCO (1966). Le fractionnenent en classe de taille a été réalisée par filtration différentielle au travers de membranes WHATMAN GF/C (porosité moyenne de $1 \mu \mathrm{m}$ ), Sartorius en nitrate de cellulose de $12 \mu \mathrm{m}$ de porosité et de nylon à bluter de $45 \mu \mathrm{m}$ de vide de maille. Les limites des trois fractions $1-12 \mu \mathrm{m}, 12-45 \mu \mathrm{m}$ et 45 - $160 \mu \mathrm{m}$ ont été choisies arbitrairement, en fonction des porosités de membrane disponibles dans le comnexce, dans la mesure où dans la littérature il n'existe pas de définition stricte de la fraction nanoplanctonique, la limite supérieure de cette classe fluctuant selon les auteurs entre 10 et $60 \mathrm{\mu m}$ (RAI, 1982 ; SICKO-GOAD et STROEMER, 1984). Le choix de la classe 12 - $45 \mu \mathrm{m}$ a étê dicté par le fait que dans la 
littérature il est admis que le zooplancton broute électivement sur les algues dont les dimensions n'excèdent pas la borne supérieure de taille retenue ici (GLIWICZ, 1977).

\section{2-4 Mesure de 1a production primaire}

La méthode adoptée est directement issue de celle décrite par STEEMANNIELSEN (1952) utilisant le ${ }^{14} \mathrm{C}$ comme traceur de I'incorporation de carbone selon un protocole expérimental décrit dans (ALEYA et al., $1988 b$ ). Après incubation ( 3 heures), des aliquots de 10 à $25 \mathrm{~cm}^{3}$ sorit. immédiatement filtrés sur des membranes de porosité identique à celle utilisée pour le dosage de 3. a chlorophylle $a$, les membranes whatman $\mathrm{GF} / \mathrm{C}$ étant remplacées par des Millipore HAWP en raison de l'utilisation d'un compteur de rađioactivité à fenêtre mince (TRACERLAB).

\section{2-5 Calcul des rapports Production/biomasse}

Si les données relatives à la biomasse et à I'activité photosynthétique des peuplements présentent un intérêt écclogique certain, en nous renseignant d'une part sux l'importance du stock de nourriture disponible pour les consommateurs de premier ordre et, d'autre part sur la quantitê de matière synthêtisée par les producteurs par unité de temps, elle ne nous apporte aucune information sur l'état physiologique de la communauté algale. C'est dans cette perspective que NAUWERCK (1963) a introduit la notion de coefficient d'activité exprimé par le rapport Production/Biomasse. Afin de rendre comparables les valeurs de ce rapport obtenues pour chacune des deux estimations de biomasse retenues, nous avons choisi d'exprimer ce paramẽtre en terme de carbone.

Pour les biovolumes transformés en terme de poids frais selon la relation $10^{6} \mathrm{\mu m}^{3}=1 \mathrm{\mu g}$ nous avors admis à la suite de la grande majoxité des auteurs, que le carbone reprếsentait 12 de ce poids-frais (STRATHMANN, 1967 ; HAMILTON et HOLM-HANSEN, 1967 ; PRIDMORE et HEWITT, 1984).

Pour la transformation en carbone des teneurs en chlorophylle $a$, la valeur du coefficient de conversion est beaucoup plus controversée (STRICKIAND, 1960). A la suite des travaux de RYTHER et al., (1971), RIEMAN et al., (1982) et DESSERY et al., (1984), nous avons retenu une valeur de $30(\mathrm{C}=$ chlorophylle $a \times 30)$. A la suite de ces transformations, les valeurs de $P / B$ seront donc exprimées en $h^{-1}$. Bien que représentant l'inverse d'un temps de renouvellement de la biomasse, ce rapport doit être considéré conme biaisé dans la mesure où la production primaire n'est assurée que durant la journée solaire et que, durant cette période, son intersité varie considérablement (et de manière non proportionnelle) avec l'intensité lumineuse. 


\section{3 - RÉSultats}

\section{3-1 Fraction de taille $<12$ um}

\section{a) Biovolumes}

La bionasse de cette fraction de taille fluctue entre 0 et $2,3 \mathrm{mg} . \mathrm{1}^{-1}$ (le 23-04, à $1 \mathrm{~m}$ ). Cette dernière valeur, liée à la présence de la Cryptophycée Cryptcmonas caudata, est cependant exceptionnelle puisque 75 des biomasses mesurées sorit comprises entre 0 et 0,2 mg..$^{-1}$ (valeur moyenne $0,12 \mathrm{mg} . \mathrm{1}^{-1}$ ), (tableau 2) (fig. 1a).

\section{b) Chlorophylie $a$}

Les concentrations en chlorophylle $a$, varient de l'état de traces (le 2-07, à $10 \mathrm{~m}$; le 23-07, au fond) à $22,5 \mu \mathrm{g} . \mathrm{I}^{-1}$ (le 23-04, à $10 \mathrm{~m}$ ), lors au développement de la chlorophycée Chlorella $\mathrm{sp.} \mathrm{(fig.} \mathrm{2a).}$

c) Production primaire

Les valeurs de l'activité photosynthétique évoluent de 0 à $24,03 \mathrm{mg} \mathrm{C} \mathrm{m}{ }^{-3} \mathrm{~h}^{-1}$ (fig. 3a). Cette dexnière valeux est à relier au développement de diverses Chlorophycées : Chlamydomonas sp., Tetraedon minimum, Chlorella sp. (Eig. 4a).

d) $P / B$ "Comptage"

Les valeurs relatives à cette fraction de taille varient de 0 à $0,2 \mathrm{~h}^{-1}$, les plus importantes étant essentiellement enreg̣istrées en été (voisines de $0,18 \mathrm{~h}^{-1}$ ) (tableau 3 ).

Etart admis que les celiules de petite taille sont douées d'une grande capacité d'assimilation photosynthétique (RAI, 1982 ; MUNAWAR et al., 1988), l'obtention de 21 \& seulement de valeurs comprises entre 0,08 et $0,2 \mathrm{~h}^{-1}$, nous semble traduire la sous estimation de la bicmasse calculée à partir des biovolumes. 66 \& des valeurs sont comprises entre 0 et 0,04 (moyenne $: 0,09 \mathrm{~h}^{-1}$ ) ( $\mathrm{fig} .5 \mathrm{a}$ ).

e) P/B "Chlorophylle a"

Bien qu'atteignant un maximurn de $0,187 \mathrm{~h}^{-1}$ le $30 / 7$ à $1 \mathrm{~m}$, les valeurs de ce rapport sont. le plus souvent voisines de $0,01 \mathrm{~h}^{-1}$ (tableau 3), 70 \& étant comprises entre 0 et $0,02 \mathrm{~h}^{-1}$ (moyenne : $0,023 \mathrm{~h}^{-1}$ ) (fig. 6a).

\section{3-2 Fraction de taille $12-45 \mu \mathrm{m}$}

\section{a) Biovolumes}

La biomasse de cette fraction de taille est impcrtante puisqu'elle varie de $0,3 \mathrm{mg} \cdot \mathrm{1}^{-1}$ (le 23-07, au fond) à $9,78 \mathrm{mg} \cdot \mathrm{1}^{-1}$ le $02-09$ à $2 \mathrm{~m}$ ) (fig. 1b).

Les plus fortes valeurs (comprises entre 4 et $9 \mathrm{mg} .1^{-1}$ ) sont obsexvées en été (du $11 / 6$ au $3 / 9$ ), dars la zone supérieure du lac. Elles coIncident avec la présence de différentes formes coloniales parmi lesquelles la Cyanophycée Gomphosphaeria lacustris et les chlorophycées Coelastrum combricum et Sphaerocystis schroeteri. 
Tableau 2.- Pourcentages moyens de la biomasse (estimée à paxtir des biovolumes et de la chlorophylle a) et de la production primaire pour les trois fractions de taille ( $\sigma$ : écart-type; m : moyenne ; c.v. : coefficient de variation).

Table 2.- Mean percentages of the three fractions biomass (expressed by biovolumes and chlor phyll a) and primary production ( $\sigma$ : standard deviation; $\bar{m}:$ average ; c.v. : variation coefficient).

\begin{tabular}{|c|c|c|c|c|c|c|c|c|c|c|c|c|c|c|c|}
\hline \multirow[b]{3}{*}{ Classes de taille } & \multicolumn{6}{|c|}{$\begin{array}{c}\text { Bio "Comptage" } \\
(\mathrm{mg} / 1)\end{array}$} & \multicolumn{6}{|c|}{$\begin{array}{c}\text { Bio "Chlorophylle" } \\
(\mu \mathrm{g} / 1)\end{array}$} & \multicolumn{3}{|c|}{$\begin{array}{l}\text { Production primaire } \\
\left.\text { (mg. } \mathrm{c} / \mathrm{m}^{3} / \mathrm{h} .\right)\end{array}$} \\
\hline & sur & $0 \mathrm{~m}$ & $-4 m$ & sur & $0 \mathrm{~m}-$ & Fond & sur & $0 \mathrm{~m}$ & $-4 m$ & sur & $0 \mathrm{~m}-$ & Fond & su & $0 \mathrm{~m}$ & $4 \mathrm{~m}$ \\
\hline & $\bar{m}$ & $\sigma$ & c.v. & $\bar{m}$ & $\sigma$ & $c . v$. & $\bar{m}$ & 0 & c.v. & $\overline{\mathrm{m}}$ & d & c.v. & $\overline{\mathrm{m}}$ & $\sigma$ & c.v. \\
\hline$<12 \mu \mathrm{m}$ & 6 & 7 & 129 & 5 & 6 & 127 & 27 & 14 & 54 & 26 & 14 & 55 & 15 & 14 & 100 \\
\hline $12-45 \mu \mathrm{m}$ & 58 & 24 & 42 & 56 & 24 & 43 & 49 & 22 & 46 & 47 & 22 & 46 & 65 & 25 & 39 \\
\hline $45-160 \mu \mathrm{m}$ & 36 & 23 & 62 & 39 & 24 & 62 & 24 & 13 & 57 & 27 & 14 & 53 & 20 & 18 & 91 \\
\hline
\end{tabular}

Tableau 3.- Valeurs moyennes des $\mathrm{P} / \mathrm{B}$ "Comptage" et $\mathrm{P} / \mathrm{B}$ "Chlorophylle" pour les trois classes de taille phytoplanctoniques. Table 3. - Mean values of $P / B$ "Counts" and $P / B$ "Chlorophyll" relative to the three fractions.

\begin{tabular}{|c|c|c|c|}
\hline $\begin{array}{c}\mathrm{P} / \mathrm{B} \text { (Comptage) } \\
\text { en } \mathrm{h}^{-1}\end{array}$ & Moyenne & Ecart-type & Coefficient de variation \\
\hline $\begin{aligned} & \text { fraction }<12 \mu \mathrm{m} \\
& 12-45 \mu \mathrm{m} \\
& 45-160 \mu \mathrm{m}\end{aligned}$ & $\begin{array}{l}0,090 \\
0,050 \\
0,026\end{array}$ & $\begin{array}{l}0,085 \\
0,040 \\
0,038\end{array}$ & $\begin{array}{r}95 \\
93 \\
145\end{array}$ \\
\hline $\begin{array}{c}\mathrm{P} / \mathrm{B}(\mathrm{Ch} \text { lorophylle }) \\
\text { en } \mathrm{n}^{-1}\end{array}$ & & & \\
\hline fraction $\begin{aligned}< & 12 \mu \mathrm{m} \\
& 12-45 \mathrm{\mu m} \\
& 45-160 \mu \mathrm{m}\end{aligned}$ & $\begin{array}{l}0,023 \\
0,047 \\
0,036\end{array}$ & $\begin{array}{l}0,034 \\
0,041 \\
0,115\end{array}$ & $\begin{array}{r}125 \\
88 \\
299\end{array}$ \\
\hline
\end{tabular}


Figure 1, abc.-Histogrammes des fréquences du pourcer:tage de la biomasse de chaque classe de taille par rapport à la biomasse totale.

Figure 1, abc.-Percentages of the three biomass size fractione frequencies tc the total biomass.
a : classe $1-12 \mu m$;
b : classe $12-45 \mu \mathrm{m}$;
c : classe $45-160 \mu \mathrm{m}$.

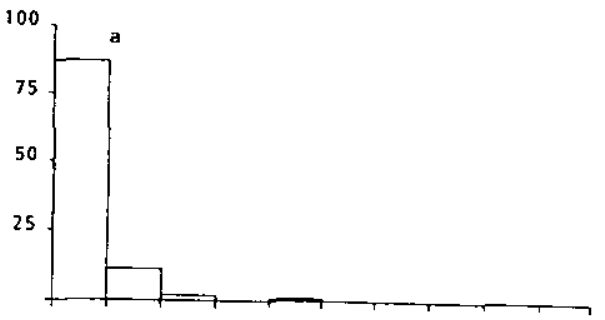

Bio./Bio. Tot.

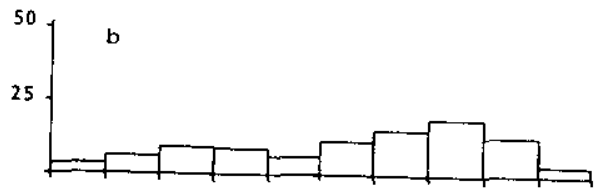

Bio./Bio. Tot.
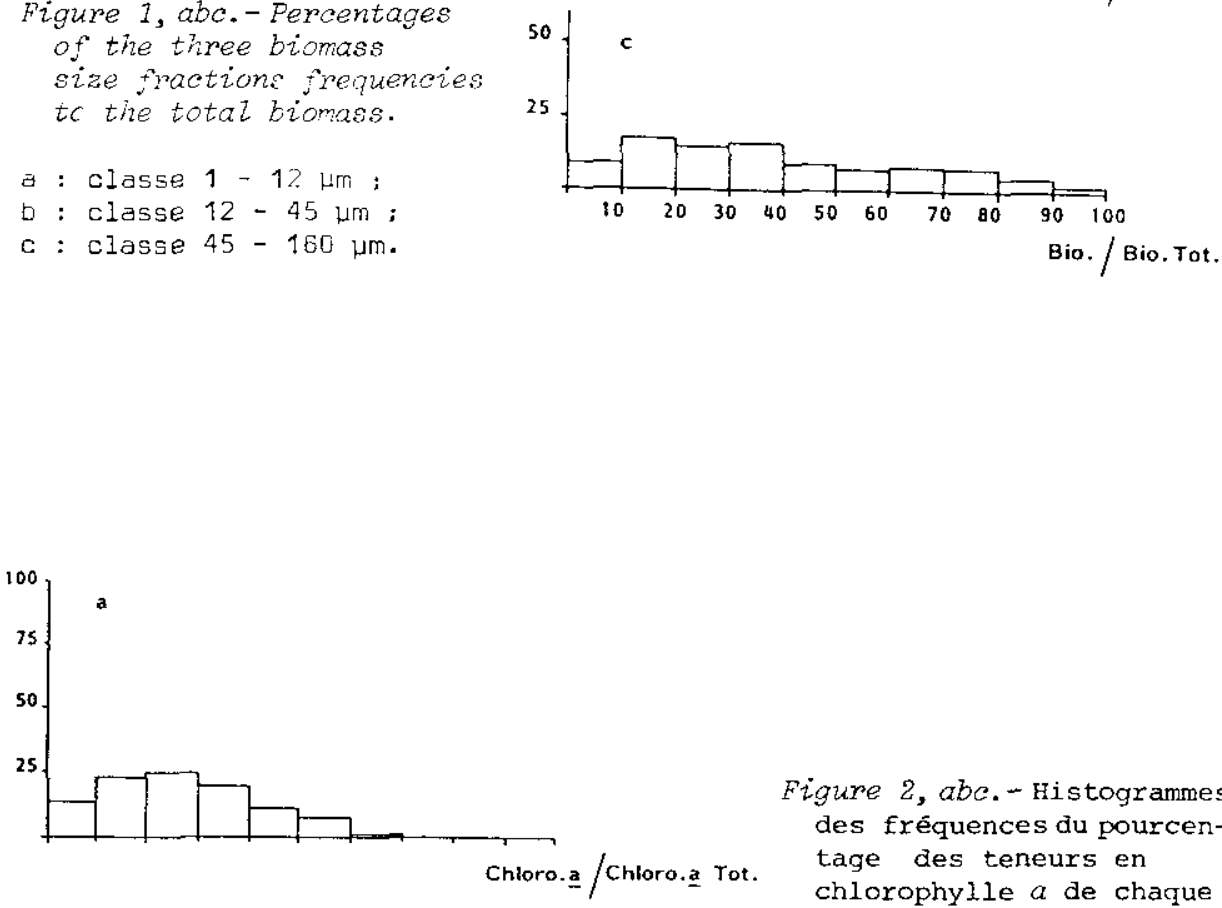

Figure 2, abc.-Histogrammes des fréquences du pourcentage des teneurs en chlorophylle $a$ de chaque classe par rapport à la chlorophylle a totale.

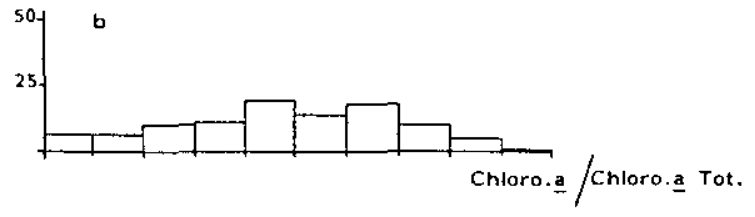

Figure 2, abc.-Percentages of the three chlorophylze a concentrations size fractions frequencies to the total chloro-

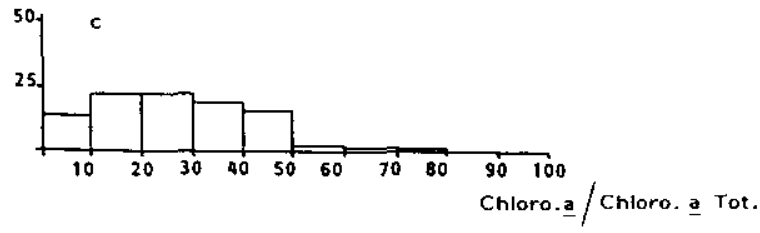
phylze a.

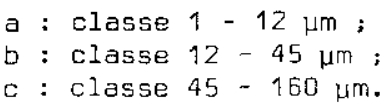

b : classe 12 - $45 \mu \mathrm{m}$; : classe $45-160$ um. 


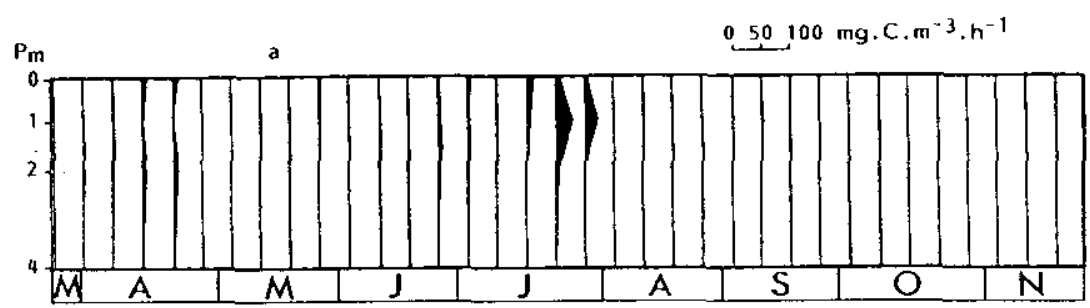

$b$

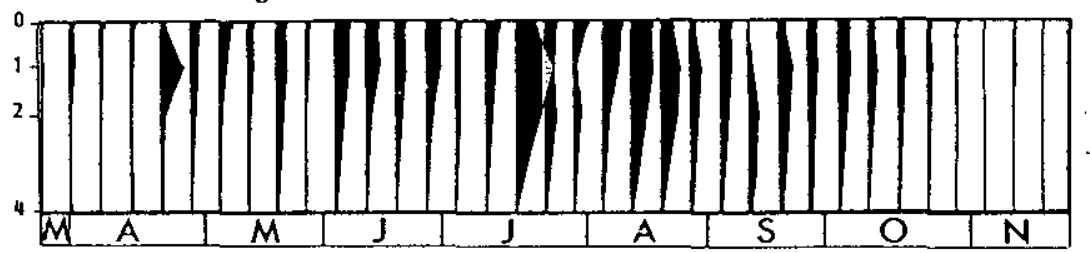

$\mathrm{c}$

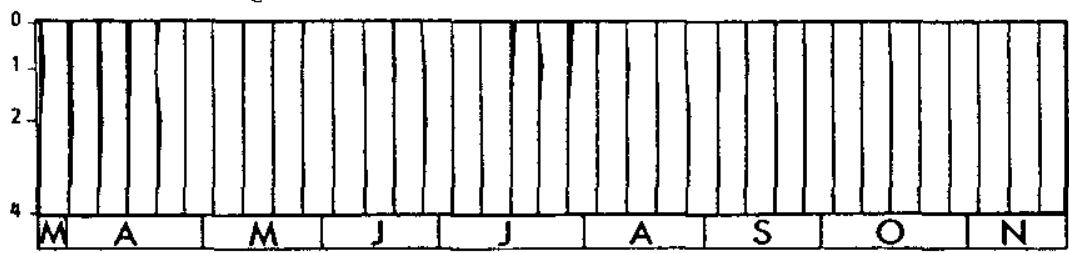

Figure 3, abc. - Profils verticaux de 1'activité photosynthétique.

Figure 3, abc.- Vertical profiles of primary production.

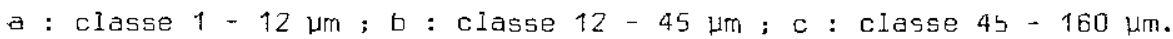

\section{b) Chlorophylle a}

Les concentrations en chlorophylle a évoluent de l'état de traces à $29,5 \mathrm{\mu g} \cdot \mathrm{1}^{-1}$ (le $16-07$, à $2 \mathrm{~m}$ ) lors du développement des Diatomées Cyclotelia psp., et des Chlorophycées S. schroeteri, C. cambricum et C. microporum (fig. 2b).

\section{c) Production primaire}

Pour cette classe de taille, l'activité photosynthétique varie de 0 (Le $8 / 10$ à $4 \mathrm{~m}$ ) à $50,58 \mathrm{mg} \mathrm{C} \mathrm{m} \mathrm{m}^{-3} \mathrm{~h}^{-1}$ (le $16 / 7$ à $1 \mathrm{~m}$ ) (fig. 3b).

Au cours de la période marsmavril, la valeur maximale $30,95 \mathrm{mg} \mathrm{C} \mathrm{m}{ }^{-3} \mathrm{~h}^{-1}$ est obtenue le $23 / 4$ à $1 \mathrm{~m}$. Celle-ci est associée au développement de Cuclotella glomerata et $C$. catenata. Fin mai, 1'activité photosynthêtique diminue pour atteindre $6 \mathrm{mg} \mathrm{C} \mathrm{m}^{-3} \mathrm{~h}^{-1}$ dans les deux premiexs mètres en raison du déclin des Diatomées précitées. A partir du mois de juin et jusqu'à la fin août. I'intensité des processus photosynthétiques s'accentue, essentiellement dans la zone $0-2 \mathrm{~m}$, atteignant la valeur maximale de $50,58 \mathrm{mg} \mathrm{C} \mathrm{m}^{-3} \mathrm{~h}^{-1}$ le $16 / 7$ à $1 \mathrm{~m}$. Cette intensification de la production primaire cos̄ncjess avec le développement dans le milieu de $S$. schroeteri, Chodatella cïizatr, $C$. cambricum et G. Zacustris (fig. 4b). 

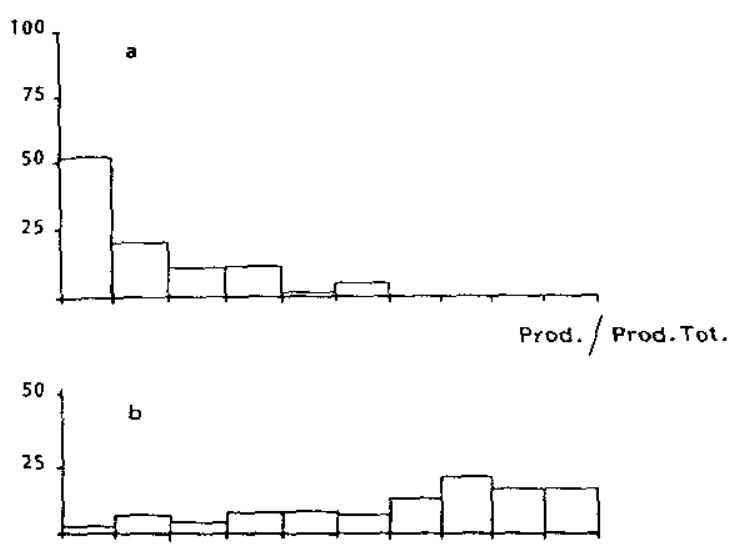

Figure 4 , abc.- Histogrammes des fréquences du pourcentage de la production primaire de chaque classe de taille par rapport à la production totale.

Prod./Prod.Tot. Figure 4, abc.-percentages

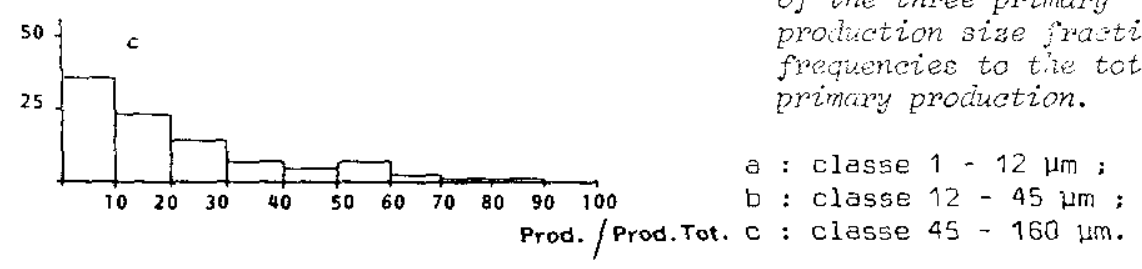
of the three primary proiliction size rastions frequencies to tive total primaxy production.

: classe $1-12 \mu \mathrm{m}$;

b : classe $12-45 \mu \mathrm{m}$;

Prod./Prod. Tot. c : classe $45-160 \mathrm{~mm}$.

Figure S. abc. - Histogrammes des frequences des valeur's des coefficients a'activi-

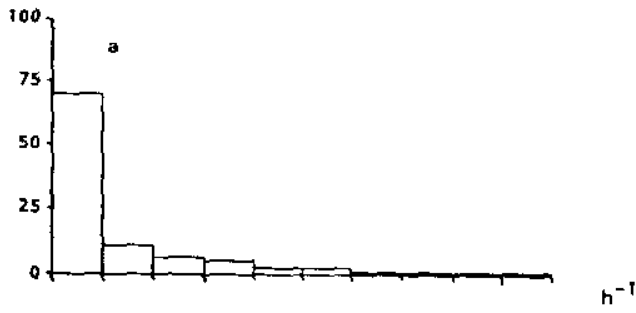
té $\mathrm{P} / \mathrm{B}$ "Comptage".

Figure 3. - Histograms of $P / B$ "Counts" frequencies values.
a : classe 1 - 12 um;
b : classe 12 - $45 \mathrm{hm}$;
c: classe 45 - 160 нm.
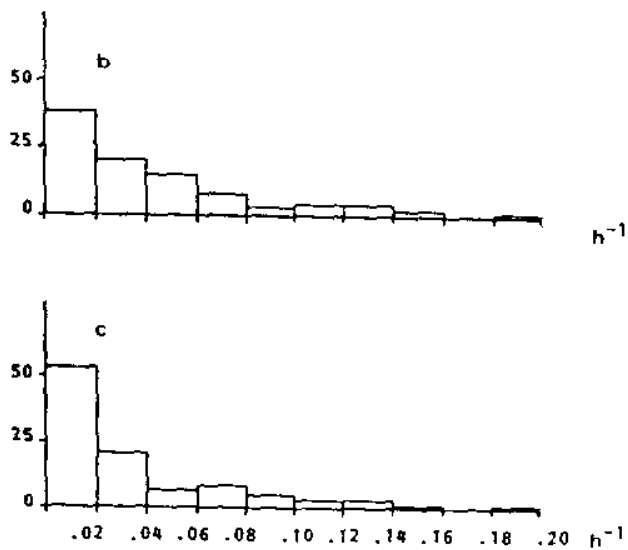

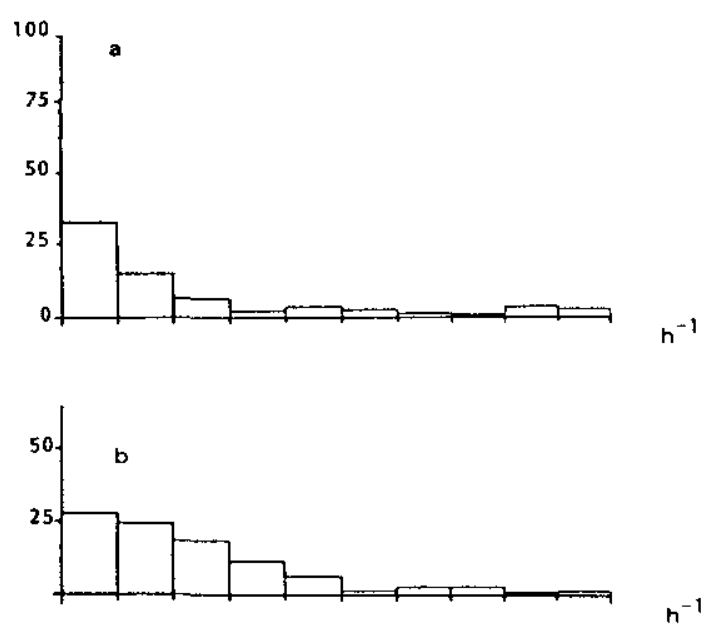

Figure 6, abc. - Histogrammes des fréquences des valeurs des coefficients d'activité P/B "Chlorophylle".

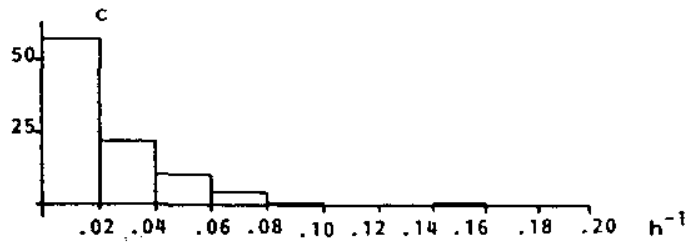

Figure 6, abc.-Histograr:a of $P / B$ "Chtorophylz" frequencies values.

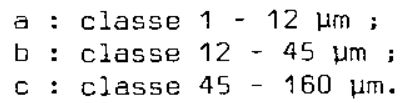

\section{d) P/B "Comptage"}

Les valeurs enregistrées varient de 0 à $0,20 \mathrm{~h}^{-1}$ (tableau 3). Ce maximum étant atteint les $17 / 9$ et $24 / 9$ à la suite du développement de G. Zacustris. D'importantes valeur's de ce coefficient d'activité sont également obtenues au printemps, $0,16 \mathrm{~h}^{-1}$ le $2 / 4$ et 0,18 le $23 / 4$, associées au développement de Cryptomonas ovata.

En été, la valeur la plus élevée enregistrée est de $0,18 \mathrm{~h}^{-1}$ le $21 / 8$ a $1 \mathrm{~m}$, lors du développement de $C$. micropomm. La moyenne des coefticients d'activité de cette classe est de $0,05 \mathrm{~h}^{-1}$. 70 o des valeurs étant comprises entre 0 et $0,06 \mathrm{~h}^{-1}$ (fig. 5b).

\section{e) P/B "Chlorophylle $a$ "}

Pour cette fraction de taille, les valeurs de ce rapport varient de 0 à $0,16 \mathrm{~h}^{-1}$ (tableau 3). Aussi bien au printemps qu'en été, les coefficients d'activité sont faibles vaxiant peu autour d'une moyenne de $0,03 \mathrm{~h}^{-1}$ exceptions faites des sorties du $2 / 4$ et du $21 / 8$ au cours desquelles ont été respectivement enregistrées des valeurs de $0,1 \mathrm{~h}^{-1}$ et $0,12 \mathrm{~h}^{-1}$.

Durant I'automne, I'élévation relative des valeurs qui atteignent $0,13 \mathrm{~h}^{-1}$ le $8 / 10$ à $2 \mathrm{~m}$ et $0,16 \mathrm{~h}^{-1}$ le $1 / 10$ à $1 \mathrm{~m}$, est essentiellement. liée à la diminution de la biomasse. 62 \& des valeurs des $P / B$ chlorophylle sont comprises entre 0 et 0,04 (moyenne : $0,047 \mathrm{~h}^{-1}$ ) (fig. $6 \mathrm{~b}$ ). 


\section{3-3 Fraction $45-160 \mathrm{~m}$}

\section{a) biovolumes}

La biomasse associée à cette classe de taille apparaît comme très variable, les valeurs extrêmes relevées étant de 0 (le 20/08, au fond) et $10,5 \mathrm{mg} \cdot 1^{-1}$ (Le $28 / 05$, en surface).

En mars et avril, c'est la Diatomée Melosira italica subsp. subartica qui représente l'essentiel de la biomasse phytoplanctonique. Par la suite, au mois de mai, ce sont les cyanophycées Anabaena flos-aquae et A. spiroïdes qui représentent les espèces dominantes.

En été et en automne, cette classe de taille ne présente que de faibles biomasses, fxéquemment inférieures à $2 \mathrm{mg} \cdot 1^{-1}$. L'espèce dominante est alors Anabaena macrospora accompagnée de Microcystis aeruginosa (fig. $1 \mathrm{c}$ ).

\section{b) Chlorophylle a}

Les concentraticns en chlorophylle a varient de l'état de traces à $20,2 \mathrm{Hg.} 1^{-1}$, ie $23 / 04$, à $10 \mathrm{~m}$, en raison de la présence simultanée d'A. flos-aquae et de M. itaíca (alors en voie de sédimentation) (fig. 2c).

\section{c) Production primaire}

Pour cette classe de taille, les valeurs de production varient de 0 à $12,18 \mathrm{mg} \mathrm{C} \mathrm{m}{ }^{-3} \mathrm{~h}^{-1}$ (fig. $3 \mathrm{c}$ ). L'essentiel de 1 'activité photosynthétique printanière se situe au mois d'avril en raison du développement de M. italica la valeur de production atteinte le 2/04 en surface est de $10,28 \mathrm{mg} C \mathrm{~m}^{-3} \mathrm{~h}^{-1}$. La fin du mois $d^{\prime}$ avril est caractérisée par des valeurs voisines de $8 \mathrm{mg} \mathrm{C} \mathrm{m} \mathrm{m}^{-3} \mathrm{~h}^{-1}$ dans la zone $0-1 \mathrm{~m}$ coincidant avec le développement d'A. flos-aquae. La plus forte valeur de production enregistrée de mai à la lère moitié de juillet est de $6,47 \mathrm{mg} \mathrm{C} \mathrm{m}{ }^{3} \mathrm{~h}^{-1}$, le 13/05 en surface. Cette valeur est concomitante du développement sporadique d'A. spirôldes.

Dès le début de la seconde moitié de juillet, on assiste à une intensification de I'activité photosynthétique avec des valeur's de 12,18 et $11,52 \mathrm{mg} \mathrm{C} \mathrm{m}{ }^{-3} \mathrm{~h}^{-1}$, en surface, respectivement les 16 et 30 juillet. Ces valeurs sont associées au développement d'A. macrcspora mais ne correspondent pas au maximum de biomasse enregistrée polir cette fraction. Le reste de l'année est. caractérisé par des valeurs faibles se situant en moyenne autour de $2 \mathrm{mg} \mathrm{C} \mathrm{m}{ }^{-3} \mathrm{~h}^{-1}$ en moyenne dans la zone $\mathrm{o}-2 \mathrm{~m}$ et principalement liées à la présence d'A. macrospora (fig. $4 \mathrm{c}$ ).

d) P/B "Comptaze"

Le ccefficient d'activité varie de 0 à $0,2 \mathrm{~h}^{-1}$. A I'exception des sorties du $17 / 09$ et du $22 / 10$ au cours desquelles est enregistrée une valeur de $0,19 \mathrm{~h}^{-1}$ en surface et à $1 \mathrm{~m}$, les coefficients d'activitê sont faibles tolit au long de l'annee, (moyenne : $0,026 \mathrm{~h}^{-1}$ ) (tableau 3), (fig. 5c).

e) P/B "Chlorophylle a"

Les valeurs de ce rapport évoluent de 0 à $0,2 \mathrm{~h}^{-1}$. Cette dernière valeur étant enregistrée le 30/07 en surface, alors que dominait $A$. macrospora (tableau 3). 53 \% des valeurs sont comprises entre 0 et $0,02 \mathrm{~h}^{-1}$ (moyenne : $0,039 \mathrm{n}^{-1}$ ) (fig. $6 \mathrm{c}$ ). 
Les résultats obtenus mettent en évidence l'importance relative majeure de la fraction de taille 12 - $45 \mu \mathrm{m}$ au sein du peuplement phytoplanctonique global du lac d'Aydat. Cette fraction représente en moyenne 47 à 58 \& de la biomasse totale selon que les estimations sont respectivement. basées sur le dosage de la chlorophylle a ou le calcul des biovolunes et assure 65 \& de l'activité photosynthétique totale. Ces résultats sont conformes à ceux rapportés par de nombreux auteurs. Ainsi RAI (1982) signale des pourcentages moyens de biomasse et d'activité photosynthétique associés à la fraction < $35 \mu \mathrm{m}$ atteigant respectivement 70 \& et 75 \% dans un lac eutrophe alors que pour ELSER et at., (1986) 40 à 90 \& de la production primaire sont assurés par la fraction $22-75 \mu \mathrm{m}$ au sein d'un lac faiblement eutrophe (tableau 2). Cette prëdominance de la fraction 12 - 45 um est confirmée par le rôle majeur qu'elle exerce sur la turbidité des eaux comme le traduit l'existence, seulement pour cette classe d'une corrélation négative hautemertt significative entre l€s teneurs en chlorophylle qui lui sont associées et lé profondeur de disparition du disque de Secchi ( $r=-0,472, p<1 \%, 33$ ddl). Ces observations corroborent les résultats de nombreux travaux mettant en éviderce l'importance des algues et des particules de petite taille dans les phénomènes d'extinction de la lumière (BACKMANn et JONES, 1974 ; HAFFNER et EVANS, 1974; LORENZEN, 1980). EDMONSON (1980), xapporte que pour une même quantité de matière, les particules de petite taille affectent davantage la transparence de l'eau que les particules de grande taille. Dans le cas du lac d'Aydat, l'absence de corrélation statistiquement significative entxe $z s$ et les teneurs en chlororphylle $a$ de la fraction < $12 . \mu \mathrm{m}$ est liée à la faible contribution relative de cette classe de taille à la biomasse phytoplanctonique totale. Deux types de causes, vraisemblablement additives sont à invoquer poirr interpréter I'existence de cette faible proportion de cellules de petite taille.

- En premier lieu, si l'on se réfère au schéma proposé par PAVONI (1963) pour relier 1 'importance relative du nanoplancton et niveau trophique des lacs, il apparait que le pourcentage moyen des teneurs en chlorophylle a associées à la classe $<12$ um mesuré à Aydat (26 \%), situe cet écosystème dans la catégorie des lacs eutrophes modérés. Ce rësultat est tout à fait conforme au statut trophique attribué au lac a'Aydat à partir de la prise en compte des paramètres classiquement utilisés en ce domaine (teneurs en éléments nutritifs, en chlorophylle $a$, assimilatjon photosynthêtique ...) (ALEYA, 1987). En fait, tous les travaux réalisés sur ce sujet mettent en évidence une importance quantitative prépondérante de la fraction nanoplanctonique en milieu oligotrophe (GLIWICZ, 1967 ; MUNAWAR et MUNAWAR, 1975 ; MUNAWAR et al., 1978 ; ROSS et DUTHIE, 1981 ; WATSON et KALFF, 1981 ; BRUNO et al., 1983 ; FAHNENSTIEL et al., 1986), importance qui diminue en milieux mésotrophe et eutrophe modếré et réaugmente en milieu eutrophe très marqué (KALFF, 1972 ; SPODNIEWSKA, 1983). Le retour à la prépondérance des cellules de petite taille dans les lacs trèr eutrophes est à rattacher aux conditions limitantes du développement du phytoplancton qui sont alors essentieliement liées à l'auto-ombrage (MUNAWAR et al., 1977. PAERL, 1977 ; SPODNIEWSKA, 1983), en raison de la relation nor linéaire qui existe entre volune cellulaire et ombrage (HARRIS, 1978), les petites cellules étant alors favorisées par leur importante teneur en chlorophylle par unité de volume. Ces études ont aussi démontré que les algues de petite dimension supportent plus facilement les conditions extrêmes que les alques de grande taille sans pour autant s'y développer de façon optimale 
étart essentiellement adaptées à résister à une grande variêté àe conditions environnementales (PAVONI, 1963 ; MUNAWAR et at., 1978). Dans la mesure où, aucun élément nutritif ne semble affecter de manière drastique le développement algal global di lac d'Aydat, il est vraisemblable que les algues de la plus petite fraction de taille $(<12 \mu \mathrm{m})$ ne tirent aucun avantage compétitif de leur rapports $S / V$ élevés qui, $s$ 'ils leur permet une meilleure exploitation des nutriments engendrent également des pertes plus importantes par respiration et excretion que les algues de grande taille (GELIN et RIPL, 1978; RAI, 1988), L'intensité de ces deux types de phénomènes étant largement dépendarte de surfaces d'échanges avec le milieu extérieur (SMITH et KALFF, 1983).

- En second lieu, il est communément admis que les zooplanctontes, généralement plus abondants en milieux eutrcphes qu'en milieux oligotrophes, exercent une pression de broutage préférentiellement orientée vers les espèces de petite taille (GLIWICZ, 1977). Tel semble bien etre le cas au lac d'Aydat où les résultats rapportés par OUIAD ALI (1988) font appaxaître $\alpha^{\prime}$ une part une contriblition majeure des Rotifères au taux de filtraticn global du peuplemert zooplanctonique et d'autre part une corrélation significative $(r=0,39 ; p<5 \%, 32$ dal) entre densité des Rotifères et activité photosynthëtique des algues de la classe $<12 \mu \mathrm{m}$. Cela semblerait traduire la simultanéité d'une activation de la production primaire nanoplanctonique par les excrétats des Rotifères et une consommation quasi immédiate, par ces herbivores, de la bjomasse ainsi formée.

Au niveau de la comparaison des valeurs moyennes des coefficients d'activité $(P / B)$ associés aux diverses fractions, nos résultats apparaissent quelque peu ambigus. En effet, il est communément admis que. les cellules de petite taille présentent l'activité métabolique la plus intense, donc les $P / B$ les plus élevés, en raison de rapports surface/ Volume: (S/V) particulièrerent importants (BANSE, 1976 ; TAGUCHI, 1976 ; PETERS, 1983 ; MURPHY et HAUGEN, 1985). Dans le même ordre d'idée $c^{\prime}$ est à l'élévaticn de la valeur moyenne du rapport $S / V$ au sein des peuplements algaux qu'est le plus souvent attribuée l'optimisation des transferts de matière et d'énergie (MALONE, 1971 ; GELIN et RIPL, 1978 ; SCHLESINGER et al., 1981 ; MALONE et NEALE, 1981 ; TAKAHASHI et BIENFANG, 1983 ; SMITH et KALFF, 1983 ; HUTTUNEN et KUPARINEN, 1986) au niveau des producteurs primaires.

Si les corxélations linéaires établies entre activité photosynthétique et valeurs de $S / V$ au sein de chacune des classes de taille considérée isolément apparaissent dans le cas de nos données comme statistiquement significatives (tableau 4) confirmant ainsi l'existence d'une relation directe entre les 2 paramètres, les valeurs moyennes de $P / B$ associées à la fraction < 12 um sont très difféxentes selon le mode d'expression de la biomasse. En effet, alors que conformément aux hypothèses généralement. admises, la hiérarchisation des valeurs obtenues pour $\mathrm{P} / \mathrm{B}$ "Comptage" met clairement en évidence une décroissance de l'activité métabolique avec l'augmentation de taille, il n'en va pas de même avec les valeurs de $P / B$ "Chlorophylle" qui apparaissent minimales pour la classe < $12 \mathrm{\mu m}$.

Deux types de causes, non exclusives sont susceptibles d'etre invoquées pour interpréter ces divergences.

- Au premier type se rattachent les divers biais introduits dans les estimations de biomasses pax les deux méthodes retenues ici. 
- En ce qui concerne le calcul des bjovolumes basé sur les dénombrements cellulaires, il est démontré par de nombreux travaux réalisés tant en lacs et en réservoirs (PAERL, 1978 ; BOSWEL et al., 1980, ELSER et al., 1986 ; ALEYA et AMBLARD, 1988) qu'en mer ou estuaires (MEL'NIKOV, 1976 ; SINCLAIR et al., 1979 ; BOOTH et al., 1982 ; DAVIS et SIEBURTH, 1982 ; CARON, 1983 ; REID, 1983) qu'ils sousestiment fortement $1^{\prime}$ importance relative de la fraction nanoplanctonique en raison :

a) d'une mauvaise préservation, de ces formes particulièrement fragiles par les fixateurs usuels tel que le Lugol utilisé ici (BLOEM et BAR GILISSEN, 1988\}.

b) d'une mauvaise sédimentation dans les chambres de comptage des cellules de petite taille.

c) d'une omission pure et simple lors des dénombrements cellulaires.

Tableau 4.- Valeurs des coefficients de corrélation de pearson obtenues entre activité photosynthétique et rapports $\mathrm{S} / \mathrm{V}$ pour les trois classes de taille.

Table 4.- Results of the correlations : prirnary production to the three fractions $s / v$ ratios.

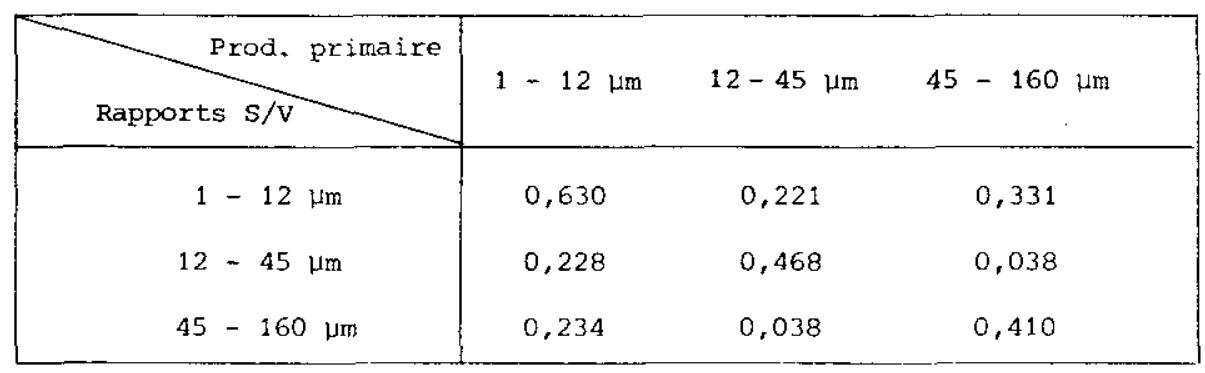

$r(p<0,05)=0,349$

$r(p<0,01)=0,449$.

ces trois sources d'erreurs, fréquenment cumulées conduisent donc dans notre cas à une sous-estimation de la biomasse nanoplanctonique et par voie de conséquence à une surestimation des valeurs de $P / B$ attribuées à la fraction < $12 \mathrm{Hm}$.

- Dans le cas des estimations basées sur le dosage des teneuxs en chlorophylle $a$, de nombreuses sources d'erreur sont également susceptibles d'être invoquées. S'il est généralement admis qu'elles assurent une meilleure prise en compte des cellules de petite taille que le calcul des biovolumes, il est toutefois reconnu que les teneurs en pigments sont, par unité de volume, supérieures pour la fractior nanoplanctcinique que pour les cellules de grande taille (MUNAWAR et al., 1978, MALONE et al., 1979 ; ELSER et al., 1986). L'adoption d'un facteur unique de conversion entrầne donc simultanément une surestimation des biomasses associées à la fraction < $12 \mu \mathrm{m}$ et une scius-estimation de celles liées à la classe 45 - $160 \mu \mathrm{m}$. Dans le cas particulier de notre étude cette dernière sous-estimation semble de surcrost amplifiée par la faible teneur en chlorophylle a des Cyanophycées, algues largement dominantes parmi les espèces retenues sur les filtres de 45 um de porosité. 
- Le second type de biais introduit dans les valeurs de $P / B$ attribués à chacune des classes de taille est inhérent à la nature des espèces rencontrées lors de la prêsente étude. En effet, la classe 45 - 160 Hm est composée icj dars sa quasi totalité par des formes coloniales (Anabaena spp., M. italica) dont les cellules présentent des rapports $\mathrm{S} / \mathrm{V}$ voisins de ceux des espèces associées à la classe de taille $<12 \mu \mathrm{m}$. Nous devons donc considérer icj, à la suite de TAKAMURA et aí., (1986) que ces espèces coloniales doivent être assimilées à des formes nanoplanctoniques.

Au terme de cette étude, il nous apparait utile de dégager un certajn nombre de conclusions quant à l'intérêt écophysicologique du fractionnement en classes de taille des peuplements phytoplanctoniques.

Au niveau des prélèvements ponctuels cette méthodologie apparaít comme très informative. En effet, il apparaist fréquemment un décalage important entre contributions à la bıomasse et à l'activíté photosynthétique selon les différentes classes de taille. Cette analyse autorise de ce fait une interprétation beaucoup plus précise que celle déduite de l'étude globale du peuplement non seulement au niveau du nrocessus successionnel mais également quant aux relations trophiques entre producteurs primaires et herbivores.

Les biais méthodologiques intrcduits tant au niveau du fractiornement que de la détermination des biomasses associée à chacune des classes de taille doivent inciter à la prudence quant à l'analyse comparative des valeurs prises par les indices d'activité métabolique tels que le rapport $\mathrm{P} / \mathrm{B}$.

\section{REMERCIEMENTS}

Nous remercions la fondation $J$. Moulin qui nous a permis l'accès au site d'Aydat. Cette étude a été réalisẻe grâce au financement accordé par le CNRS dans le cadre du GRECO 81, le PIREN (ASP eau) et la Région Auvergne.

\section{RÉFÉRENCES BIBLIOGRAPHIQUES}

ADMIRAL W., VENEKAMP L.A.H. (1986). Significance of Tintinnid grazing during blooms of Phaeocustis Pouchetii (Haptophyceael in Dutch coastal waters. Weth. J. Sea Res., 20(1): 61-66.

aleya l., DEVAuX J., Magotiri h., MaRValin O., AMBLARD C. (1988a). Usefulness of the use of simultaneous methods for the estimation of phytoplankton biomass. Eur. J. Protistol., 23: 334-342.
ALEYa L., DEVauX J., MaRValin o. (1988b). Production primaire et activité bactérienne hétérotrophe potentielle au sein d'un lac eutrophe (lac d'Aydat, Puy-deDome, France). Rev. Sci. Ecu, 1: 23-35.

ALEYA L., DEVAUX J., (1989) . Relations entre la transparence de 1 'eau et les teneurs en chlorophylle $a$ de trois fractions de taille phytoplanctoniques $d$ ' un lac eutrophe (lac d'Aydat). Ann. Sc. Vat. Zoo2., Paris, 9: 257-262. 
ALEYA L., AMBLARD C. (1988). Importance quantitative de diverses fractions de taille phytoplanctonigues en milieu lacustre eutrophe. Hydrobiologia (sous presse).

ALEYA L. (1987). Dynamique des populations phytopianctoniques du lac d'Aydat 'Pay-ieDôme, Eroncel. Bi-masse et activité métabolique de diverses fractions de tailie. Thèse Doct. 3ène cycle. Univ. Clemont, 11,216 pp.

BACLMANN R.W., JONES J.R. (1974). Phosphorus input and algal blooms in lake. Fow State. J. Res., 49: 155-160.

BANSE K. (1976). Rates of growth, respiration and photosynthesis of uniceliular algae as related to cell size review. $v$. Phycol., 12: 135-140.

BERGQUIST A.M., CARPENTIER S.R., LATINO J.C. (1985). Shifts in phytoplankton size structure and community composition during grazing by contrasting zooplankton assemblages, Eimol. Oceanogr., 30: 1037-1C45.

BIENFANG P.K. (1985). Size structure and sinking rates of various microparticulate constituents in oligotrophic Hawailan waters. Mar. Ecot. Prcg. Ser., 23: 143-151.

BLOEM J., BÂR GILISSEN M.J.B. (1988). Fixing nanoflagellates. Arch. Hyoirobiol. Beih. Emgeb́n. Limnol., 31: 275-280.

BOOTH B.C., LEWIN J. NORRIS R.E. (1982). Nanoplankton species predominant in the subartic Pacific in May and June 1978. Deep Sea Res., 29: 185-200.

BOSWELL J.T., PERRY W.B., STANEORD J.A. (1980). Analysis of Plankton dynamics in a southwestern U.S.A. reservoir using ATP assays. Int. Rev. Gesamtem dydrobiol., $65(1)$ : $155-167$.

BRUNO S.E., STAKOR R.D., SHARMA G.M. TURNER J.T. (1983). Primary productivity and phytoplankton size fracticn dominance in a temperate North Atlantic estuary. Estuaries, 6: 200-211.

CARON D.A. (1983). Technique for enumeration of heterotzophic and phototrophic nanoplankton, using epifluorescence microscopy, and comparison with other procedures. Appl. Environ. Microbiol., $46(2): 491-498$.

CRAIG S.R. (1984). Productivity of algal picoplancton in a small meromictic lake. Verh. Int. Ver. Limmol., 22: 351-354.

DAVIS P.G., SIEBURTH J.M. (1982). Differentiation of the photosynthetic and heterotrophic populaticas of nanoplankters by epifluorescence microscopy. Ann. In:t. Ocemorr., paris, 58 suppl.
DESSERY S., ILANCELOT C., BILLEN G. (1984) . Primary production and its fate in the storage basin of Mery-sur-oise. Verh. intermat. Verein. Limmol., 22: 1504-1509.

DRENNER R.W., MUMMERT J.R., DENOYELLES F. , KETTLE D. (1984). Selective particle ingestion by a filter-eeeding tish and its impact on phytoplankton corgmunity structure. Limmo:. Ocecrogn., 29: $941-948$

EDMONSON W.T. (1980). Secchi dish and chlorophyll. Limnoi. Jcecnser., 25: $378-379$.

ELSER J.J., ELSER M.M., CARPENTER S.R. (1986). Size fractionation of algal chlorophyll, carbon fixation and piosphatase activity : relaticasnip with species-specific size distributions and zooplarikton community structure, $j$. Plankton Res., 8: 365-383.

FAFNENTIEL G.L., SICKO-GOAD I., SCAVIA D. , STOERMER E.F. (1986). Importance of picoplankton in Lake superior. Can. $\therefore$. Eish. Aquat. Sci., 43: 235-240.

FINLAY B.J., BERNINGER U.G. (1984) Coexistence of congeneric ciliates (Karyorelictica : Lorodes) in relation to food resources in two freshwater lakes. J. Anim. Ecoi., 53: 929-943.

GATES M.A. , LEWG U.T. (1984). Contribution of cillated protozod to the planktonic biomass in a series of ontario lakes : quantitative estimates and dynamical relationships. J. Ptankion Res., 6: 443-456.

GELIN C., RIPL W. (1978). Nutrient decrease and response of various phytoplankton size fractions following the restauration of lake Trumen, Sweden. Arch. Hydrobiol., 81: 339-367.

GLIWICZ Z.M. (1977). Food size selection and seasonal succession of filter-feeding zooplankton in a eutrophic lake. Ekol. Pol., 25: 179-225.

HAFFENER G.D., EVANS J.H. (1974). Relation of light penetration to particle distribution in vertically mixed lacustrines environments. Br. Phycol. J., 9: 261-267.

HAMILTON R.D., HOLM-HANSEN O. (1967). Adenosine triphosphate content of marine bacteria. Limnol. Oceanogr., 12(12): 319-324.

HARRIS G.P. (1978), Photosynthesis, productivity and groweh : the physiological ecology of phytoplankton. Arch. Hydrobiol., Bech. Ergeb. Limmol., 10: 1-171. 
HERBLAND A., BOUTETLLER LE. A. (1981). The size distribution phytoplankton and particulate organic matter in the Equatorial Atlantic Ocean : importance of ultraseston and consequences. $J$. Plonkton. Res., 3: 650-665.

HEWES C.D., HOLM-HANSEN O. (1983). A method for recovering nanoplankton from filters for identification with the microscope : the filter-transfer-freeze (FTF) technique. "imnol. Oceconogr., 28: $389-394$.

HUTTUNEN M., KUPARINEN J. (1986). Species succession and productivity of ultraphytoplankton in the pelagic off Tvarminne SW coast of Finland. Opheiia., Suppl. 4: 73-83.

KALFF J. (1972). Netplankton and nanoplankton production and biomass in a North temperate zone laxe. Limnol. Ocecnogr., 17: 712-720.

IAWS E.A. (1975). The importance of respiration lossess in controling the size distribution of marine phytoplankton. Ecology, 56: 419-426.

LEGENDRE L., WATS W.o. (1971-1972). On a rapid technic for plankton enumeration. Ann. Inst. Oceanogr. Paris WL VIII, $173-177$

LOHIMAN H. (1908). Untersuchungen zur Feststeliung des volistandigen Gehaltes des Meeres an Plankton. Wiss. Meeresunters, Abt. Kiel N.F., 10: 132-170.

LORENZEN M.W. (1980). Use of chlorophyllsecchi disk relationships. Limmol. Oceanogr., 25: 371-372.

LYNCH M. , SHAPIRO J. (1981). Pzedation enrichment and phytoplankton comounity structure. Limnot. Ocecnogr., 26: 86-102.

MAC CAULEY E., KALFF J. (1980). Empirical zelationships between phytoplankton and zooplankton biomass in lakes. Can. $J$. Fish. Aquat. Sci, 38: 458-463.

MALONE T.C. (1971). The relative importance of nanoplankton and netplankton as primary procuders in Tropical Oceanic and neritic phytoplankton communities. Limmol., Decanogr., 16: 633-639.

MALONE T.C., CHEVRIN M.B., BOARDMANN D.C, (1979). The effects of $22 \mu \mathrm{m}$ screens on size-frequency distribution of suspended particles and biomass estimates of phytoplankton size fracticns. Limmol., Oceonogr., 24: 956-960.

MALONE T.C. (1980). Algal size in Morrís I. (ed.). The Physicological Ecalogy of the phytoplonkton. University of california Press, Berkeley, 433-463.
MALONE T.C., NEALE P.J. (1981). Parameters of light-dependent photosynthesis for phytoplankton size fractions in temperate estuarine and coastal environments. Mar. Biol., 61: 289-297.

MARGALEF R., ESTRADA M. (1987). Synoptic distribution of summer microplankton (Algae and Prctozoa) across the principal front in the western Mediterranean. Inv. Pesq., 51(1): 121-140.

MEL'NIKOV N.S. (1976). Conparisor on the magnitude of the microplankton biomass determined from ATP and by direct microscopy. Oceanology, 16: 181-183.

MILIERIOUX G. (1976). Contmioution $a$ l'étude du lac d'Aydat. Thèse Doct. 3ème cycle. Uriv, Clermont II, 107 pp.

MUNAWAR M. et MUNAWAR I.F. (1975). The abundance and signiflcance of phytoflagellates and nanoplankton in the St-Iawrence Great lakes. I. Phytoflagellates. Verh. Int. Ver. Limmol., 19: $705-723$.

MUNAWAR M., MUNAWAR I.F., GULP L.R., DUPUIS G. (1978). Relative importance of nanoplankton in Lake Superior phytoplankton bjomass and community metabolism. J. Great Lakes Res., 4: 462-480.

MUNAWAR M. et MUNAWAR I.F. (1986). The seasonality of phytoplankton in the North American Great Lakes, a comparative synthesis. Hydrobiologia., 138: 85-115.

MUNAWAR M. , MUNAWAR I.F., MCCARTHY L. a. (1988). Seasonal succession of phytoplankton size assemblages and its ecological implications in the North American Great Lakes. Verh. Intermat, Verein. Limnol., 23: 659-671.

MURPHY L.S., HAUGEN E.M. (1985). The distribution and abbundarce of phototrophic ultraplankton in the North Atlantic. Limol. Oceconogr., 30: 47-58.

NAUWERCK A. (1963). Die beziehungen zwischen zooplankton und Phytoplankton im See Erken. Symp. Bot. Upsalienses., 17(5): 1-163.

OULAD ALI H. (1988). Role et importance des Rotiferes planctoniques dans un lac eutrophe, le lac d'Aydat (Erance). Activités de broutage et efficacité d'assimilation. These Univ. Universite, Blaise Pascal, 225 pp..

PAERL. H.W. (1978). Effectiveness of various counting wethods in detecting viable phytoplankton. N,Z.J.Mar. Freshuater Res., 12: 67-72. 
PAERL H.W., MACKENZIE L.A. (1977). A comparative study of the diurnal carbon fixation patters of nanoplankton and net plankton. Limmol., Oceanogr., $22: 732-738$.

PAVONI M. (1963). Die Bedeutung des Nanoplankton. Scineitz. Z. Hyçroz., 25: $219-341$.

PLATT T., DENMAN K. (1977). Organization in the pelagic ecosystem. He?goimier Meereswiters., 30: 575-581.

PETERS R.H. (1983). The ecological implications of body size. Carisinige iniv. Press., Cambridge, 329 p.

POURRIOT R. (1982). Ecologie du plancton des eaux continentales. Collection $a^{\prime}$ Ecologie $n^{\circ} 10^{\circ}$, Masson ed. Paris, 198 pp..

PRIDMORE R.D., HEWITT J.E. (1984). Chloropyll $a$ as an indicator of phytoplaniton cell volume in 12 lakes, North island. New-Zealand. i. 2. $\therefore$. $\$ 0 \%, 22(2)$ : 295-301.

RAI H. (1982), Primary production of various size fractions of natural phytoplankton communities in a north German lake. Arch. Hudroiziol., 95(1-4): $395-412$.

RAI H. (1988). Activity of resdizatory electron transport system (ETS) in different size particles as a measure of carbon losses from primary producers. Verh. Internat. Verein. Limmol, 23: 503-504.

REID F. M. H. (1983). Biomass estimation of components of the marine nanoplankton and phytoplankton by the Uthermolh settling technique. I. Ptankton Res., 5: $235-252$.

REIMANN B., SQNDERGAARD M., SCHEIRUP H. H., CHRISTENSEN G., HANSEN J., NIELSEN B. (1982). Carbon netabolin during a spring diatom bloom in the eutxophic Lake mossoe. Int. Rev. Gesanten. Hydrobiol. $67(2):$ 145-185.

REYNOLDS C.S. (1984). Phytoplankton periodicity : the interactiors of forms, functions and environmental variability. Fresinater. Biol., 14: 111-142.

ROSS P.E., DUTHIE H.C. (1981). Ultraplankton biomass, productivity and efficiency in Lac Matamec, a precambian shield lake. J. Phycol., 17: 181-186.

RYTHER J.H., MENZEL D.W., HULBURT E.M., LORENZEN C.J., CORWIN N, (1971). The production and utilization of organic matter in the peru coastal current. Inv. Pesq., 35: 43-60.
SCHLES INGER D.A., MOLOT L.A., SHUTER B.J. (1981). Specific growth rates of freshwater algae in relation to cell size and light intensity. Con. J. Fish. jiquat. Sci., 38: 1052-1058.

SCOR-UNESCO (1966). Determination of phytoplankton pigments in sea water. Scor-inesco., 69 pp..

SHUTER B.J. (1978). Size dependence of phosphorus and nitrcgen subsistence quotas in unicellular organisms. iimnoi. oceonogr., 23: 1248-1255.

SICKO-GOAD L., STROEMER E.F. (1984). The need for uniform terminology concerning phytoplankton cell size fractions and examples of picoplankton from the Laurentien Great Lakes. J. Great Lakes Res., 10: 90-93.

SIEBURTH M., SMETACEX V., LEN2 J. (1978) pelagic ecosystem structure : heterotropic components of tint plankton and their relationship to plankton size-fractions. Eimot. Oceconogr., 23: 1256-1263.

SINCLAIR M.E., KEIGHAN E., JONES J. (1979). ATP as a mesure of living phytoplankton carbon in estuaries, $j$. Fish. Res. Bd. Can., 36: 180-186.

SMAYDA T.J. (1970). The suspension and sinking of phytoplankton in the sea. Oceconogr. Mar. Biol. Am. Rev., 8: 353-414.

SMITH R.E. H., KALFF J. (1982). Sizedependent phosphorus uptake and cell quota in phytoplankton. J. Phycoi., 18: $275-284$.

SMITY R.E.H., KALFF J. (1983). Competition for phosphorus among-co-occuring freshwater phytoplankton. Limnot. Oceanogr., 28: 448-464.

STEELE J.H., FROST B.W. (1977). The structure of plankton communities. Philos. Trans. R. Soc. Lond., B 280: 485-534.

SPODNIEWSKA I. (1978). Phytoplankton as the indicator of lake eutrophication. I. Summer situation in 34 Masurian lakes in 1973. Ekol. Pol., 26: 53-70.

SPODNIEWSKA I. (1979). Phytoplankton as the indicator of lake eutrophication. II. Summer situation of 25 Masurian lakes in 1976. Ekol. Pol, 27: 481-496.

SPODNIEWSKA I. (1983). ECological characteristics of lakes in North-Eastern Poland versus their trophic gradient. vI. The phytoplankton of 43 lakes. Eckol, Pol., 31(2): 353-382, 
SPRULES G., MUNAWAR M. (1986). Plankton size spectra in relation to ecosystem productivity, size, and perturbation. Con. J. Fish. Aquat. Sci., 43: 1789-1794.

STEEMANN-NIELSEN E. (1952), The use of radio-active carbon $\left(C^{14}\right)$ for measuring organic prcduction in the sea. J. Cons. Explor. Mer., 18: 117-140.

STOCKNER, J.G., ANTIA N.J. (1986). Algal picoplankton from marine and freshwater ecosystems : a multidisciplinary perspective. Con. J. Fish. Aquat. Sci, 43: 2472-2503.

STRATHMANN R.R. (1967). Estimating the organic carbon content of phytoplankton from cell volume of plasma volume. Limnol. Oceconogr., 12(3): 411-418.

STRICKLAND J.H. (1960). Measuring the production of marine phytoplankton. Butl. Fish. Res. Board Can., 122, $172 \mathrm{pp}$.
TAGUCHI S. (1976). Relationship between photosynthesis and cell size of marine diatons. J. Physiol., 12: 185-189.

TAKAHASHI M. BIENFANG P.K. (1983). Size structure of phytcplankton biomass and photosynthesis in subtropical Hawalian waters. Mar. Biol., 76: 203-211.

TAKAMURA N., IWAKUMA T., MASAYUKI Y. (1986). Photosynthes is of size-fractionated phytoplankton populaticn in hypertrophic lake Kasumugaura. jazan. Arch. تucirobiol. , $113,235-257$.

UTHERMOHL H. (1958). Zur Vervollkomnung der quantitative phytoplanixon-Methodik. Mitt. Interm. Ver. Eimmoi., 9: 1-38.

WATSON S., KALFF J. (1981), Relationships between nanoplankton and lake trophic status. Con. J. Fish. Aquat. Sei., 38: 960-967. 